\title{
Les défis du repreneuriat forestier : Étude de cas de relève familiale selon une approche systémique
}

\author{
France Asselin ${ }^{\mathrm{a}}$, Étienne St-Jean ${ }^{\mathrm{b}}$ et Luc LeBel
}

\section{INTRODUCTION}

Dans la plupart des pays industrialisés, une proportion élevée des entreprises est appelée à être transmise au cours des $\mathbf{1 0}$ prochaines années, compte tenu des nombreux départs à la retraite prévus des propriétaires.

Dans la plupart des pays industrialisés, une proportion élevée des entreprises est appelée à être transmise au cours des 10 prochaines années, compte tenu des nombreux départs à la retraite prévus des propriétaires ${ }^{1}$. Le secteur forestier québécois de la récolte vit aussi ce phénomène de départ à la retraite des propriétaires de $\mathrm{PME}^{2}$. Pour l'économie québécoise et canadienne, la reprise des entreprises forestières est identifiée comme vecteur de la transmission du savoir-faire en matière de récolte et d'opérations forestières, ce savoir étant névralgique pour l'industrie ${ }^{3}$. Notre travail poursuit la documentation du repreneuriat dans ce secteur amorcé par Audet (2008) auprès des entrepreneursaspirants non nécessairement impliqués encore dans le processus de reprise. Il aborde les défis de la relève entrepreneuriale impliquée dans un processus repreneurial ainsi que les moyens utilisés pour relever ces défis avec succès. Pour y parvenir, nous présentons une brève revue de littérature sur les principaux défis et facteurs influençant le processus de repreneuriat. Par la suite, nous soulevons les particularités du secteur forestier, énonçons nos questions de recherche et décrivons la méthodologie privilégiée pour nos travaux. Après un bref descriptif des cas étudiés nous présentons nos résultats. Une discussion sur les enjeux particuliers de la relève forestière vient clore cette communication.

\section{Pour l'économie québécoise et canadienne, la reprise des entreprises forestière est identifiée comme vecteur de la transmission du savoir-faire en matière de récolte et d'opérations forestières, ce savoir étant névralgique pour l'industrie.}

\section{RECENSEMENT DES ÉCRITS : LES DÉFIS DU REPRENEURIAT}

Il existe trois types de relève, dont la distinction se fait selon la relation entre le repreneur et le cédant : la relève familiale provenant de la famille du cédant, la relève interne constituée des employés de l'entreprise et la relève externe, sans lien familial ou d'emploi.

La reprise et la transmission d'entreprises sont deux appellations qui évoquent le même processus, mais réfèrent à deux angles différents, soit l'angle du repreneur (i.e. celui qui reprend) ou l'angle du cédant (i.e. celui qui transmet). Le terme repreneuriat, la conjonction des mots reprendre et entreprendre, est utilisé également pour désigner ce processus ${ }^{4}$. Il existe trois types de relève, dont la distinction se fait selon la relation entre le repreneur et le cédant : la relève familiale provenant de la famille du cédant, la relève interne constituée des employés de l'entreprise et la relève externe, sans lien familial ou d'emploi. Le processus consiste en deux transferts, soit celui de la propriété et du leadership. Ce processus est influencé par plusieurs acteurs ${ }^{5}$. En premier lieu, les acteurs de l'environnement interne, soit le repreneur, le cédant, l'entreprise (employés, administrateurs ou actionnaires) et la famille. En second lieu, il y a aussi, les acteurs de l'environnement externe ce qui comprend les parties prenantes du macro-environnement de même que les accompagnants sur le processus 
(humain) ou sur le contenu (aspects techniques et financiers). Une modélisation du processus de reprise adapté à ces trois types de reprises le décompose en cinq étapes : 1) l'amorce, 2) la mise en œuvre, 3) la transition, 4) l'officialisation et 5) l'après-transfert ${ }^{5}$.

Selon l'angle du cédant, plusieurs défis sont communs aux différents types de relève se situent principalement à trois niveaux : 1) la planification de la relève, 2) les aspects financiers et techniques (économique et légal), et 3 ) les aspects humains et relationnels ${ }^{6}$. En prenant l'angle du repreneur, nous traitons ci-après des défis humains de la préparation, des défis relationnels et finalement des défis financiers. Puisque nous nous intéressons aux transferts familiaux, l'accent sera mis sur ces recherches en particulier.

\subsection{Les défis de la préparation}

\subsubsection{Le repreneur : caractéristiques recherchées et préparation}

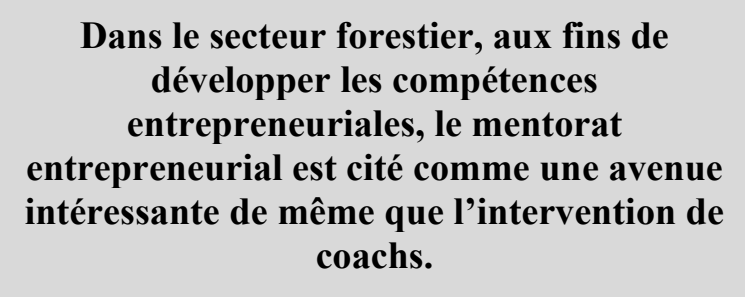

La préparation du repreneur est identifiée dans plusieurs travaux comme un facteur significatif influençant la réussite de la reprise ${ }^{5}$. Lors de l'examen du parcours de développement des repreneurs d'entreprises québécoises, il fut constaté que $75 \%$ des repreneurs ayant réussi ont réalisé des études postsecondaires ${ }^{8}$. Aux fins de reprises par l'interne, ressemblant à la reprise par l'interne, regroupe selon trois types les compétences à acquérir soit les compétences techniques, les connaissances pratiques (et explicites) du fonctionnement de l'entreprise et du secteur, et les compétences entrepreneuriales. L'importance de réussir à transmettre « l'entrepreneurship » du propriétaire en place est soulevée, cette compétence représentant parfois " la » compétence distinctive de la PME reprise ${ }^{9}$. Dans le secteur forestier, aux fins de développer les compétences entrepreneuriales, le mentorat entrepreneurial est cité comme une avenue intéressante de même que l'intervention de coachs ${ }^{3}$.

\subsection{Les défis relationnels de la reprise}

\subsubsection{L'influence du cédant}

Le rôle du cédant est prépondérant aux fins de la préparation de la relève familiale ${ }^{10}$ (Cadieux, 2007). En repreneuriat familial, la qualité de la relation entre le cédant et le repreneur est déterminante, car contributive à la transmission des valeurs. Le dialogue et la proximité entre le cédant et le repreneur favoriseraient la qualité de relation entre eux. Toutefois, il est fréquent d'observer une difficulté chez le cédant à se désengager de la direction. Son accompagnement peut faciliter son lâcher-prise ${ }^{7}$.

\subsubsection{L'influence des acteurs internes et de l'état de l'entreprise elle-même.}

Les résultats d'une étude pancanadienne comptant des repreneurs familiaux et non familiaux soulignent l'importance de la planification pour faciliter l'acceptation du repreneur par l'interne pour maintenir l'harmonie avec les employés et dans la famille. Compte tenu de l'attachement à l'ancien dirigeant, pour être accepté, le repreneur doit gérer avec doigté le changement qu'il représente $^{4}$. De plus, l'état de l'entreprise au moment de la reprise influencerait aussi le niveau de résistance des employés face au changement de dirigeant (Deschamps et Paturel, 2005, p.217).

Les résultats d'une étude pancanadienne comptant des repreneurs familiaux et non familiaux soulignent l'importance de la planification pour faciliter l'acceptation du repreneur par l'interne pour maintenir l'harmonie avec les employés et dans la famille.

\subsubsection{L'influence des acteurs externes à l'entreprise}

Il est essentiel que le repreneur démontre de la crédibilité auprès des tiers avec lesquels l'entreprise entretient des relations d'affaires aux fins de réussir sa reprise. Les accompagnants, autres acteurs externes, interviennent parfois dans 
le processus. Les coachs, experts en management et les psychologues interviennent notamment au niveau de la gestion du changement, de la gestion des relations et pour faciliter la gestion du système famille par rapport à la transmission ${ }^{4,5}$.

\subsubsection{L'influence de la famille}

Pour les repreneurs familiaux, le transfert du management se révèle d'une difficulté beaucoup plus grande que pour les repreneurs non familiaux. À cet effet, les interventions des consultants peuvent représenter un atout aux succès des reprises. La présence d'un conseil indépendant favorise, semble-t-il, l'acceptation du repreneur par les autres membres de la famille.

\subsection{Les défis financiers de la reprise}

\subsubsection{Le repreneur et sa capacité d'apport financier}

Reprendre une entreprise implique souvent une mise de fonds du repreneur. Ses avoirs sont la source de financement la plus citée par les repreneurs canadiens de tous types ayant participé à l'étude de FCEI en 2005, soit pour $50 \%$ d'entre eux ${ }^{1}$. Par contre, les défis relatifs à la mise de fonds seraient moindres pour les repreneurs familiaux ${ }^{8}$. La relève d'entreprises forestières de récolte vivrait un défi de financement particulièrement important en comparaison à d'autres types d'entreprises; pour une micro-entreprise de récolte employant moins de 5 personnes, les actifs requis pour opérer peuvent représenter une valeur 1,5 million et il n'est pas encore question de fonds de roulement ${ }^{2}$.

\subsubsection{L'influence du cédant}

Selon l'étude canadienne de la FCEI, les cédants sont souvent des acteurs importants dans le financement puisque $30 \%$ des repreneurs de tous types sont financés par eux. Par ailleurs, pour les familiaux, plusieurs cédants facilitent les reprises à leur progéniture via des dons ou en leur consentant des rabais. Par contre, certains cédants rendent plus difficile le transfert de propriété. À ce sujet, les études font état de surprises désagréables sur la fiabilité des données financières ${ }^{13,11}$.

\subsubsection{L'influence de l'entreprise, valeur et dimension}

La difficulté d'évaluation serait le deuxième obstacle aux reprises, après celle du financement du projet. Lorsque les entreprises à reprendre et leurs comparables ne sont pas publiques, il est effectivement plus difficile de les évaluer ou de comprendre la "comptabilité créative " des vendeurs ${ }^{12}$.

\subsubsection{L'influence des acteurs externes (fournis- seurs de financement particulièrement)}

Les travaux de chercheurs français soulignent, toujours en regard de la reprise de la propriété, que l'un des principaux freins aux reprises serait les banques "frileuses » ${ }^{4}$. Au Québec, il est évoqué des résultats de recherche que « le monde financier, en général, n'est pas prêt pour la relève. Des programmes existent et sont affichés par les institutions financières, mais ils ne concernent peu ou pas les transactions pour les entreprises qualifiées de modestes ${ }^{11}$.

\subsubsection{L'influence des accompagnants}

Au-delà des comptables qui participent parfois aux évaluations ou effectuent minimalement un due diligence, d'autres spécialistes ont été recensés comme contribuant à l'établissement du prix, soit à surmonter un des principaux défis financiers de la reprise. En effet, une recherche québécoise, s'intéressant au rôle des consultants, rapporte des cas où le fiscaliste ou le courtier en vente d'entreprises ont contribué à déterminer un prix de vente, rapprochant ainsi les parties opposées d'une même famille ${ }^{16}$.

\subsubsection{L'influence de la famille}

En général, et tel qu'énoncé précédemment, la reprise dans la famille est généralement plus facile compte tenu de dons ou de prix réduits consentis. Des cas relevés par Pinard témoignent et démontrent à quel point l'établissement du prix peut comporter une lourde charge émotive et provoquer de graves conflits au sein des familles, ces conflits pouvant même nuire à la santé de l'entreprise. De plus, la perception de l'équité peut devenir une barrière sérieuse à une reprise harmonieuse et comprendre les écarts de perception serait contributif à une reprise familiale harmonieuse $\mathrm{e}^{11,14}$. 


\section{LE SECTEUR FORESTIER : LES ENTREPRISES DE RÉCOLTE}

L'industrie forestière est névralgique pour l'économie canadienne et est un pilier de son économie rurale. Depuis le début des années 2000, l'industrie forestière vit une crise qui fut qualifiée, par le premier ministre Jean Charest, de " la pire crise de l'histoire du secteur forestier québécois ». De plus, l'industrie forestière a un impact significatif sur l'économie des régions et tel que déjà indiqué au début de cet article, cette industrie est aussi touchée par l'approche de la retraite d'un grand nombre de ses propriétaires dirigeants ${ }^{15}$.

Les PME effectuent, au Québec, la majorité de la récolte des forêts publiques, celles-ci constituant $80 \%$ de l'exploitation. Il est donc clair que ces PME constituent un maillon névralgique de la chaine de valeur au sein de l'industrie forestière.

$\mathrm{Au}$ Québec, la ruralité et les intérêts des industriels et, plus tard, la mécanisation ont contribué à l'élaboration d'une organisation économique de la récolte forestière effectuée principalement en sous-traitance par des PME. Les PME effectuent, au Québec, la majorité de la récolte des forêts publiques, celles-ci constituant $80 \%$ de l'exploitation. Il est donc clair que ces PME constituent un maillon névralgique de la chaine de valeur au sein de l'industrie forestière ${ }^{16}$.

Les particularités de ce secteur industriel seraient à même d'influencer considérablement les reprises dans ce secteur. À cet effet, en sus de la forte mécanisation du secteur forestier, s'ajoutent aussi les coûts élevés des équipements. Drolet décrit les entreprises de récoltes forestières comme ayant " un chiffre d'affaires annuel médian de $550000 \$$, une valeur moyenne des actifs de $600000 \$$ et un nombre d'employés se situant autour de quatre ». Conjugué à la crise que connaît l'industrie et à l'incertitude des contrats alloués aux entrepreneurs forestiers, le financement d'une reprise pourrait être un enjeu crucial pour les repreneurs de cette industrie ${ }^{17}$.

\section{QUESTIONS DE RECHERCHE ET MÉTHODOLOGIE}

Considérant les défis de la reprise et des facteurs d'influence recensés et préoccupés par le repreneuriat familial au sein des entreprises québécoises de récolte forestière, nous avons énoncé comme suit nos questions de recherche. Q1) Comment les repreneurs se préparent-ils à reprendre une entreprise forestière de récolte? Et parmi les acteurs du système, qui et comment contribuent-ils à leur développement? Q2) Quels sont les défis relationnels relatifs à la prise de leadership d'une entreprise de récolte forestière? Et parmi les acteurs du système, qui et comment influencent-ils la nature de ces défis et contribuent-ils à ce qu'ils soient surmontés? Q3) Quels sont les défis financiers auxquels sont confrontés les repreneurs d'entreprises forestières de récolte? Et parmi les acteurs du système, qui et comment influencent-ils la nature de ces défis et contribuent-ils à ce qu'ils soient surmontés?

Nous cherchions d'abord et avant tout à documenter et à explorer un phénomène. En conséquence, nous avons adopté l'approche qualitative. De plus, nous avons opté pour l'étude de cas ${ }^{19}$. Les entreprises recherchées furent celles où le processus de reprise était déjà amorcé et où, idéalement l'étape du règneconjoint ${ }^{5}$ avait été atteint et dont le transfert de propriété était amorcé. Celles-ci furent recensées via un échantillonnage de convenance ${ }^{20}$. Aux fins de mieux pointer les conditions selon lesquelles le processus se réalise et faire ressortir les similitudes et les différences, nous avons analysé ces cas selon l'approche de la comparaison intersite ${ }^{21}$.

Les données ont été collectées lors d'entrevues semi-dirigées par téléphone, d'une durée variant d'une à deux heures en utilisant un questionnaire d'entrevue semi-structurée. Deux répondants par cas ont été interviewés séparément, soit le père propriétaire et un représentant de sa relève. Ceci constitue une triangulation des observations, avec l'objectif d'en augmenter la validité et d'en enrichir la documentation du phénomène, en ayant minimalement la perspective du cédant et d'au moins un repreneur. 


\section{DESCRIPTION DES CAS ÉTUDIÉS}

\subsection{Cas Tremblay (nom fictif)}

À la fin des années 1990, M. Tremblay (père), alors âgé de 50 ans, est propriétaire de TRR, une compagnie de récolte forestière devenue inopérante suite à l'incendie récent des équipements. Avant de relancer TRR en utilisant les indemnités d'assurance reçues pour les équipements incendiés, le père a demandé à ses deux fils ainés s'ils étaient intéressés à se joindre au projet de relance. Puisque les fils étaient d'accord, la relance de l'entreprise a été effectuée en 2000 , le père ayant pour objectif de la leur transmettre ultimement. En 2000, le père est demeuré actionnaire unique et ses fils ont d'abord travaillé chez TRR en tant qu'employés. À ses débuts, en 2000, l'entreprise opère un ensemble d'équipements de base, soit une abatteuse et un transporteur. Un deuxième ensemble d'équipements est acheté et mis en opération dans cette première année de relance. En 2002, le père gèle la valeur de ses actions participantes et cède $22 \%$ de celles-ci à chacun de ses deux fils (pour un transfert total de $44 \%$ ). En 2004, il procède de la même manière en cédant à chacun $11 \%$ d'actions additionnelles. À la fin de 2008, lorsque les deux fils ainés sont âgés de 29 et 32 ans, ils achètent le reste des actions appartenant à leur père, celui-ci se retirant et n'intervenant plus dans les affaires de l'entreprise à partir de ce moment. Pour ce cas, lors de l'entrevue, le processus était donc complété puisqu'il se situait à l'étape de l'après-transfert. Fait à souligner, immédiatement après le rachat complet de l'entreprise par les fils, celle-ci comptant deux ensembles d'équipements et une dizaine d'employés, elle a été divisée en deux afin que chacun puisse opérer sa propre entreprise individuellement. Ainsi, l'un des fils poursuit les affaires de TRR et son frère, le répondant à cette recherche, a démarré une nouvelle compagnie après avoir acheté la moitié des actifs à son frère.

\section{RÉSULTATS}

\subsection{Les défis la préparation}

\subsubsection{Les compétences techniques}

A) Formation académique. Trois des quatre repreneurs ont suivi des cours spécifiques leur procurant des compétences liées au métier de la récolte et à l'entretien des équipements. Notamment, M. Gélinas-fils (répondant), a complété un DEP

\subsection{Cas Gélinas (nom fictif)}

M. Gélinas (père) est seul actionnaire d'une entreprise de récolte forestière. À la fin des années 1990, l'entreprise vit des difficultés financières, les opérations cessent et des équipements sont retournés aux fournisseurs ou sont liquidés. Deux de ses fils, l'un âgé de 17 ans et l'autre de 19 ans, qui travaillaient alors pour l'entreprise, sollicitent le père pour qu'il relance les opérations de récolte en s'associant avec lui. Le père, alors âgé de 40 ans, accepte la proposition et une nouvelle compagnie démarre en 2000, nommée ici " RGG ». Au démarrage, le père détient $50 \%$ des parts, chacun des fils détient $25 \%$ et les décisions sont prises en collégialité. Le père a l'intention ultime de transmettre l'entreprise à ses fils, mais le moment et la manière n'ont pas été discutés jusqu'à maintenant. Le travail est réparti ainsi en 2000 : le père et les fils opèrent les équipements et effectuent l'entretien et un employé complète l'équipe. Actuellement, l'entreprise opère un seul ensemble d'équipements. Pour ce cas, la mise en œuvre du projet de reprise a été réalisée en même temps que le démarrage de l'entreprise. Le transfert de la propriété est actuellement stationnaire à l'étape de la " mise en œuvre ", considérant que le partage de l'actionnariat est une invitation claire à reprendre, que les successeurs sont identifiés et intéressés et que l'intention du père de transmettre à ses fils est manifeste. Le transfert de direction, quant à lui, oscille entre les étapes de « transition » et «d'officialisation », étant donné que jusqu'à il y a quelques mois, le père laissait la direction de l'entreprise à ses fils (caractéristique de l'étape de transition) et le client principal ne s'adressait plus à lui, mais plutôt à ses fils. Toutefois, puisque le client principal a interrompu ses activités il y a quelques mois, le père s'est alors impliqué à nouveau dans les activités de négociation et de sollicitation auprès de la clientèle.

(diplôme d'études professionnelles) en soudagemontage, et M. Tremblay-fils (répondant), cumule pour sa part deux DEP, l'un en mécanique industrielle et l'autre en abattage et façonnage.

B) Coaching (entrainement) spécialisé sur les compétences du métier. Comme alternative de 
formation additionnelle, certains repreneurs ont bénéficié de coaching technique personnalisé et formel, directement offert sur le terrain. Spécifiquement, M. Tremblay (père), suite à l'achat d'une nouvelle " tête " pour son abatteuse multifonctionnelle, a investi 25000 \$ pour former ses fils. Ainsi, un ingénieur les a entrainés à temps plein pendant le premier mois d'opération. Son mandat était de favoriser l'expérimentation, quitte à briser des composantes. En effet, il importait pour ce père de développer chez ses fils les connaissances qu'ils ne détenaient pas luimême, mais aussi leur inculquer débrouillardise et autonomie.

C) Compagnonnage. Le compagnonnage informel a aussi contribué au développement de tous ces repreneurs répondants et ce, en étant en contact avec les mécaniciens de leurs fournisseurs. Ces derniers les conseillent souvent à distance pour résoudre des problèmes, ce qui leur permet d'apprendre. Les pères également effectuent du compagnonnage pour transférer leurs compétences du métier de la récolte et des opérations des équipements ce, jusqu'à leur niveau personnel de plafonnement en matière de savoir technologique (électronique). Malgré qu'ils en soient venus à surpasser leurs pères au niveau technique, les fils reconnaissent bel et bien l'apport majeur de leurs pères sur les fondements de leur apprentissage.

\subsubsection{Les compétences pour réussir en récolte et diriger}

Savoir produire efficacement et savoir produire de la qualité sont des compétences qui ont été identifiées par les répondants comme essentielles pour assurer minimalement la survie de leur entreprise.

A) La gestion de la productivité et de la qualité. Savoir produire efficacement et savoir produire de la qualité sont des compétences qui ont été identifiées par les répondants comme essentielles pour assurer minimalement la survie de leur entreprise. En effet, elles sont nécessaires pour que l'entreprise soit rentable, qu'elle conserve sa clientèle et qu'elle évite des pénalités pour des problèmes de non-qualité.
B) Les compétences de gestion générales. Pendant l'étape de la transition, où règnent conjointement les pères et les fils, des réunions de gestion contribuent au développement des compétences en gestion pour les deux cas observés. Ces réunions sont parfois formelles (le comptable peut être invité), parfois informelles. Par ailleurs, la capacité à adopter des stratégies adaptées de gestion des équipements serait cruciale pour la réussite d'une entreprise d'opération forestière. Cette capacité à prendre des risques mesurés (c.à-d. investir de façon préventive) est acquise au contact du cédant, en observant ses bons et mauvais coups, mais aussi via l'expérimentation. Le fils Gélinas souligne entre autres que l'accumulation des erreurs qu'il a commises sur des équipements usagés lui a permis d'acquérir cette compétence. De plus, au niveau de la gestion générale de l'entreprise, l'observation des erreurs du père dans ses affaires a elle aussi participé à développer ses compétences de gestion, et ce, selon les dires du père et du fils Gélinas.

C) La mobilisation du personnel. Dans le cas Tremblay, le père et le fils interrogé considèrent que l'exercice du leadership auprès des employés est une compétence indispensable pour les mobiliser. Le père mentionne d'ailleurs que son fils a plus de talent que lui à ce niveau, qu'il sait toujours garder son calme. Selon lui, il donne de la rétroaction de façon claire, respectueuse et en privé.

\subsection{Les défis humains de nature relationnelle}

\subsubsection{L'influence du cédant}

Préparer sa relève s'appuierait en partie sur les compétences du cédant à transmettre son savoir. $M$. Tremblay (père) a indiqué avoir su développer les compétences chez sa relève par ses conseils, en étant dur et exigeant parfois, ainsi qu'en l'observant et en la félicitant à l'occasion. M. Gélinas (père) allait dans le même sens, en ajoutant même avoir donné de tels conseils à un opérateur et que celui-ci est maintenant devenu entrepreneur.

D'après les propos recueillis, nous observons également que les deux pères ont eu une influence sur la perception qu'ont leurs fils en regard de la nature intéressante du travail d'entrepreneur en 
récolte. En effet, les pères ont exposé très tôt (dès la petite enfance) leurs fils aux activités de coupes en forêt et les fils comme les pères rapportent que cette exposition a influencé l'intérêt pour les fils à l'égard du travail en forêt. M. Gélinas indique même qu'un parent qui veut transmettre son entreprise doit d'abord s'assurer de communiquer sa propre passion à son enfant. Cette exposition a porté fruit; le fils Gélinas dit pratiquement en ces mots... qu'il est bien en forêt, qu'il ne veut plus en sortir! Le fils Tremblay indique également que l'observation de son père, qui possède une autonomie dans son travail et une certaine réussite financière, l'a inspiré dans son choix de carrière. Nous avons observé également que le contexte du travail en forêt, forçant la grande proximité des pères et des fils lors des soirées dans les camps forestiers, se prête particulièrement bien au développement des compétences de gestion via les rencontres où ils discutent de leur planification et trouvent ensemble des solutions aux différents problèmes.

\subsubsection{L'acceptation des employés}

Comme l'entreprise du cas Gélinas ne possède qu'un seul employé, cela a éliminé bien des possibilités de difficultés. Par contre, l'entreprise du cas Tremblay possédait une dizaine d'employés tout au long du processus de transition, ce qui a complexifié le défi relationnel d'être accepté par ceux-ci. En fait, les employés n'ont pas tout de suite accueilli la relève comme patron. L'officialisation par le père, auprès des employés, à l'effet que ses fils étaient devenus copropriétaires et exerçaient comme lui le rôle de patron, a été un moyen fort positif pour corriger leurs attitudes réfractaires à observer les ordres des fils et pour contrer les commérages et les jalousies. En effet, une fois cette communication effectuée, rapporte $\mathrm{M}$. Tremblay père, le climat de travail s'est amélioré. Le leadership de la relève a également contribué à l'acceptation une fois l'entreprise rachetée par les fils Tremblay. Par exemple, peu après l'annonce de leur reprise finale de la propriété, certains employés ont tenté d'obtenir des avantages additionnels. Les fils Tremblay n'ont pas plié aux demandes et ces employés ont décidé de quitter l'entreprise. Suite à leur départ, le climat de travail était meilleur au sein de l'équipe.

\subsubsection{L'acceptation des clients}

Relativement à l'acceptation des clients, il peut d'abord être rapporté qu'avant de procéder au rachat final, les fils Tremblay ont rencontré le client principal. Ils se sont assurés de son engagement à poursuivre les affaires avec eux audelà de leur reprise finale. La crédibilité qu'ils avaient su acquérir auprès de ce client pendant le retrait progressif du père a été contributive à leur acceptation. De plus, lors de conversations informelles, le client avait manifesté au père sa confiance envers les fils, qu'il considérait comme de bons travailleurs. Bien que les Gélinas soient encore dans la période de transition, il est possible d'observer que cette période sert à mettre en place la crédibilité de la relève auprès des clients. Le père notamment, en se retirant des opérations, a laissé ses fils intervenir directement avec les représentants du donneur d'ordre principal. Les fils ont alors bâti leur propre crédibilité.

\subsubsection{L'acceptation des fournisseurs}

Par rapport à l'acceptation des fournisseurs, la crédibilité de TRR (cas Tremblay) étant déjà établie avant le rachat final. Le fils Tremblay qui a poursuivi ses opérations sous le nom TRR n'a eu aucune difficulté à obtenir du crédit chez eux. Par contre, son frère qui a démarré une nouvelle compagnie en achetant la moitié des équipements, a eu des difficultés au moment de l'ouverture des comptes chez les fournisseurs. Ainsi, pour ce cas, un repreneur familial assurant la continuité était avantagé par rapport à celui qui, pour reprendre, démarre une nouvelle entreprise.

\subsubsection{La dynamique familiale}

Nous comparons ici l'impact des valeurs familiales sur l'étape post-reprise et sur la durée de l'étape transition. Chez les Tremblay, les relations familiales ont eu une incidence sur l'avènement d'une réorganisation dans la période de l'après-transfert. En effet, tel que mentionné précédemment, les frères préféraient scinder l'entreprise en deux. De longue date, le père s'attendait à ce que ses fils prennent chacun leur voie lorsqu'il ne serait plus dans l'entreprise. Il anticipait que ses fils auraient pu avoir des divergences d'opinions. La division de l'entreprise a été ici un moyen permettant d'éviter 
d'éventuels conflits entre frères et un moyen répondant aussi à leur besoin de diriger seul. Aucun intervenant externe ne fut requis pour gérer les relations dans la famille dans cette situation de réorganisation.

D'autre part, les interactions humaines dans la famille Gélinas semblent influencer le fait que la reprise ici ne soit pas terminée dans cette famille. Le père et ses fils partagent l'idée que le travail en famille est une force : "Quand tu es trois de la même famille, c'est plus fort (pour réussir en affaire) qu'un gars tout seul » dira le fils. Son père tient aussi des propos similaires. Tous sont donc satisfaits du mode de direction de l'entreprise où les décisions sont prises en collégialité. Il n'y a pas de proaction des fils incitant le père à leur vendre ses actions. De plus, le père n'est pas à l'âge de la retraite, il a un peu plus de 50 ans seulement. Aucun des membres de la famille n'aborde encore le sujet d'une transaction finale de reprise dans cette famille. Les propos tenus par les membres du cas Tremblay témoignent d'un esprit de compétition entre les enfants, contrairement aux membres de la famille du cas Gélinas. Les difficultés en affaires qu'a vécues le père Gélinas ont sans doute contribué à souder les liens familiaux pour assurer la réussite de l'entreprise. Au lieu de diviser la famille, ces expériences l'ont rapprochée. Ils ont expérimenté la réussite par l'entraide et veulent continuer selon ce modèle.

\subsection{Les défis financiers}

\subsubsection{L'influence des repreneurs et cédants}

Pour les deux cas ici rapportés, les pères ont préparé à l'avance la transmission de la propriété, en partageant très tôt l'actionnariat avec leurs fils. En effet, pour le cas Tremblay, dont le transfert de propriété s'est achevé fin 2008 , le père donnait $50 \%$ de ses actions participantes à ses deux fils ( $25 \%$ chacun) dès 2002, soit deux ans après la relance de RTT. En 2004, il leur donnait déjà une autre tranche de $22 \%$ (11\% chacun). Dans le cas de M. Gélinas, dès le démarrage en 2000, il leur a donné chacun $25 \%$ des actions participantes sans aucune compensation financière en retour.

\subsubsection{L'influence de l'entreprise et du secteur où elle se situe}

Le contexte forestier est très particulier de par l'importance des investissements qu'il requiert pour démarrer en affaires. Chaque ensemble vaut facilement plus d'un million de dollars. Les frères Tremblay, bien que relativement jeunes, disposaient de cet apport minimal pour acheter, compte tenu du fait qu'ils avaient déjà des actions. N'eût été cette préparation du transfert de propriété par gel successif d'actions et le don du père, tout nous porte à croire que cette reprise aurait été plus difficile à réaliser. Leur père évoque d'ailleurs qu'en l'absence d'une telle aide, cela se serait avéré presque impossible de reprendre l'entreprise de récolte. Par contre, cette stratégie de préparation exige que le cédant accepte de moins s'enrichir, tel qu'évoqué par M. Tremblay (père).

\subsubsection{L'influence des parties prenantes}

Les fils Tremblay n'ont bénéficié d'aucun programme ou de subvention facilitant le rachat des actions du père en 2008. Et, malgré la mise de fonds que représentaient les actions qu'ils détenaient, ils ont dû consentir à des endossements personnels majeurs pour racheter. Ils disent avoir obtenu facilement du financement dans la mesure où des garanties personnelles substantielles étaient données à la banque. Les fils Gélinas, quant à eux, perçoivent l'accès au financement comme étant plus ardu dans le contexte difficile de l'industrie des dernières années ce qu'ils appuient par le refus récent des institutions financières à les financer pour renouveler leurs équipements.

\subsubsection{L'influence de la famille et des consultants}

Dans le cas Tremblay, où le comptable a fourni les informations financières, les fils connaissaient déjà la teneur et la fiabilité des états financiers puisqu'ils étaient actionnaires. L'entreprise a été vendue sans valeur d'achalandage, ce qui semble propre au secteur et facilite d'ailleurs l'établissement du prix. De plus, les parties se montraient accommodantes entre elles. Le fils évoque n'avoir pas été agressif afin que le prix soit plus bas. Les fils, comme leur père, considèrent que le prix négocié était correct, ni trop bas, ni trop haut. Le père recherchait un prix correct, car il voulait avoir la capacité financière d'aider éventuellement son fils cadet, ses frères ainés ne voulant pas l'associer dans cette transaction. L'analyse de ces cas suggère des conclusions et pistes d'action sur trois plans : soit sur le plan de la préparation, sur le plan relationnel et sur le plan financier, ce que nous abordons à présent. 


\section{PISTES D'ACTION ET CONCLUSION}

\subsection{La préparation}

\section{Au sein de l'industrie forestière, il y a bel et bien une relève familiale qui se prépare de longue date et qui relève le défi de se préparer à l'entrepreneuriat de récolte.}

À travers les propos recensés, nous pouvons constater qu'au sein de l'industrie forestière, il y a bel et bien une relève familiale qui se prépare de longue date et qui relève le défi de se préparer à l'entrepreneuriat de récolte. Elle a su acquérir les compétences clefs de différents ordres (techniques, entrepreneuriales, propres au secteur) ce qui favorise sa capacité à devenir entrepreneur et autonome. Nous avons aussi observé que différents moyens ont contribué pour ces cas à relever le défi de la préparation dont la formation académique, le compagnonnage, le coaching, et ce, avec le support de différents acteurs du système reprise. Il nous est aussi apparu que les fournisseurs, dans l'industrie de la récolte, jouent un rôle important en regard de la relève entrepreneuriale à plus d'un égard. Ils sont recensés comme acteur mettant la relève externe en relation avec des entreprises potentiellement à reprendre ${ }^{3}$ et, comme nous l'avons observé, ils exercent, pour la relève familiale, un rôle au niveau du transfert des compétences techniques (compagnonnage et coaching spécialisé).

Le père propriétaire joue aussi un rôle prépondérant par rapport à la préparation de la relève.

Tout comme la littérature traitant de la relève familiale le soulève, le père propriétaire joue aussi un rôle prépondérant par rapport à la préparation de la relève. Nous observons que la nature artisanale du travail de récolte forestière au sein de ces petites entreprises qui fait que les propriétaires sont très impliqués dans les opérations, accentue l'importance du compagnonnage par le père sur les compétences techniques, en sus des autres rôles qu'il assume dans le transfert des compétences entrepreneuriales et de gestion. De plus, la proximité qu'impose la vie dans les camps forestiers, particularité de la récolte en forêt publique, a été favorable au transfert des pères vers les fils des compétences de gestion et de celles propres au secteur. La longue période de codirection observée pour ces cas est elle aussi particulièrement propice au transfert de compétences en gestion d'une entreprise forestière. La littérature invoque l'importance de la préparation. Même si cette préparation est faite de façon parfois informelle, les faits collectés nous permettent de constater que les pères s'y impliquent tôt et y portent une grande attention.

Le rôle qu'ont exercé les pères en socialisation en bas âge de leur fils sur la nature intéressante du travail de récolte, a influencé sans aucun doute leur intérêt pour le secteur. Cela confirme, du moins pour ces deux cas, l'influence de la socialisation primaire aux valeurs du cédant, incluant sa passion pour le travail en forêt. Nos résultats laissent penser que cette socialisation (du père ou de la mère) pourrait être particulièrement importante dans des secteurs industriels où l'attraction de la relève pose problème, par exemple en agriculture ${ }^{22}$.

Par ailleurs, le fait que les pères évoquent qu'ils sont parfois dépassés par la technologie suggère de recommander à la relève de continuellement renouveler ses apprentissages afin de conserver à jour ses compétences techniques. L'investissement dans la formation de pointe de l'ordre de $25000 \$$ de la part de M. Tremblay nous indique à quel point cette formation est coûteuse, mais aussi à quel point elle a une valeur importante aux yeux des cédants pour la préparation de la relève familiale. Il est évident que si la valeur perçue n'avait pas été si grande, M. Tremblay n'aurait pas fait un tel investissement. La spécificité du secteur fait en sorte qu'une formation personnalisée semble être parfois l'unique façon d'accéder aux connaissances techniques de pointe requises. Aussi, considérant les coûts directs et indirects importants de cette formation de pointe et de sa nécessité, il peut être recommandé que les gouvernements et organismes paragouvernementaux soutiennent et sensibilisent les entrepreneurs du secteur et souvent même leurs 
associés-relève, dans la mise en place de ce type d'accompagnement.

Nous observons que les fils sont assez peu en lien avec le développement des affaires. Compte tenu de l'évolution qui pourrait survenir suite à la crise forestière, il est possible que la relève soit davantage exposée à des activités stratégiques de promotion de leurs services ou de négociation. Il y aurait lieu de proposer, dans le cadre de la formation à la relève entrepreneuriale, certains éléments de négociation, de stratégie et de vente, éléments que les entrepreneurs actuels ont eu moins à développer, compte tenu de leur contexte. Nous pensons que toute formation complémentaire destinée aux repreneurs d'entreprise de récolte forestière doit tenir compte des disponibilités, des saisons et aussi du fait que les repreneurs sont souvent éloignés de la maison et des grands centres. Les moyens technologiques de communication à distance et la e-formation sont des avenues à considérer, de même que la flexibilité des accompagnants de toute nature qui travailleront à leur développement.

\subsection{Le plan relationnel}

\section{Sur le plan relationnel, l'arrivée en poste de nouveaux dirigeants est facilitée \\ lorsqu'une certaine gestion du changement est assurée tout comme l'indiquait la littérature consultée.}

Notre étude nous a permis d'observer que sur le plan relationnel, l'arrivée en poste de nouveaux dirigeants est facilitée lorsqu'une certaine gestion du changement est assurée tout comme l'indiquait la littérature consultée ${ }^{4}$. Ainsi, l'officialisation du transfert par le cédant auprès des employés est une stratégie qui, pendant la transition, a facilité l'acceptation de la relève. Nous avons observé que les défis augmentent avec le nombre d'employés. Finalement, bien que la littérature indique que certains accompagnants peuvent faciliter la gestion des relations dans la famille, nous n'avons observé que les cas n'avaient pas éprouvé le besoin de requérir de tels services.

Il a aussi été possible de constater que le repreneuriat peut être à l'origine d'entreprises essai- mées, particulièrement lorsque les entrepreneurspères ont plusieurs enfants et que leur entreprise est facilement divisible. Les donneurs d'ouvrage et aussi les fournisseurs devraient être sensibles à cette réalité et pourraient d'ailleurs intervenir pour faciliter cette transition lorsqu'il s'agit d'essaimage. Les donneurs d'ouvrage pourraient même sensibiliser les entrepreneurs forestiers à la préparation de leur propre relève et, au besoin, faciliter l'essaimage. Les fournisseurs pourraient également se montrer plus ouverts quant aux normes exigées pour consentir du crédit aux entreprises essaimées.

\subsection{Le plan financier}

Sur le plan financier, nous avons observé que la difficulté à fixer le prix d'une entreprise de récolte ne semble pas importante, bien qu'établir le prix soit recensé comme une difficulté importante dans la littérature (Bruce et Picard, 2005). Le cas de vente finalisé démontre que la valeur des équipements est relativement facile à établir entre membres d'une même famille qui connaît bien l'industrie. Le cas démontre aussi que les petites entreprises de récolte, du moins dans la famille, peuvent se transiger sans valeur d'achalandage, ce qui résulte en un prix correct pour toutes les parties et facilite la fixation du prix.

Nos résultats font état que les dons d'actions des pères à leurs fils ont été des stratégies qui ont grandement facilité l'accès à l'entrepreneuriat pour cette relève. Ceci nous porte à croire que cette façon de faire peut être transposée dans un contexte non familial où des entrepreneurs s'associeraient progressivement à des employés prometteurs avec l'objectif de leur vendre la totalité des actions à terme.

Par ailleurs, aucun programme n'a facilité le rachat aux frères Tremblay et ils ont dû donner des garanties personnelles importantes. Il est possible aussi que le monde financier n'offre pas de produits adaptés aux reprises de petites entreprises forestières pour les PME en général. Si tel est le cas, afin de faciliter les reprises, les intervenants financiers et instances gouvernementales devraient éventuellement revoir les produits de financement qu'ils destinent aux entrepreneurs qui désirent reprendre une entreprise existante, notamment les repreneurs familiaux. 


\section{Conclusion}

\section{La cohabitation bigénérationnelle est une force favorisant non seulement la réalisation des reprises mais sert parallèlement au succès de l'entreprise elle- même.}

Les deux cas de relève examinés proposent, par le récit de leur expérience du processus, plusieurs stratégies qui ont mené à la réalisation et au succès entrepreneurial de cette relève. De plus, nous constatons que certains facteurs positifs sont associés au contexte, dont la proximité entre pères et fils, fort propice au mentorat du père lorsque père et fils travaillent ensemble en forêt et vivent dans les camps forestiers. Les recommandations qui découlent de nos travaux invitent les acteurs de l'industrie à s'impliquer dans le succès du repreneuriat pour tous les types de relève. Cela pourrait favoriser la préparation de la relève et le transfert du savoir-faire précieux des entrepreneurs de cette industrie.

Nous constatons, en trame de fond de nos entrevues, que la cohabitation bigénérationnelle est une force favorisant non seulement la réalisation des reprises mais sert parallèlement au succès de l'entreprise elle-même. En effet, l'engagement de la relève porte main forte aux entrepreneurs en place de par leur qualité d'engagement à l'égard $\mathrm{du}$ succès de l'entreprise pendant tout le processus de relève. Tous les acteurs du système bénéficient de leur valeur ajoutée, et ce, avant même que ne soient complétés les deux transferts.

\section{BIBLIOGRAPHIE}

${ }^{1}$ Bruce, D. « La relève des PME: mise à jour » document de recherche, Fédération canadienne de l'entreprise indépendante (FCEI), 2006, p.1-6. Bruce, D. et Picard, D. «La relève: la clé de la réussite » document de recherche, Fédération canadienne de l'entreprise indépendante (FCEI), 2005, p. 1-15. Mandl, I. Problems in Transfer of Business and Successions in Austria, Institute for SME research, 2004. Mass Mutual Financial Group/Raymond Institute, American Family Business Survey, janvier 2003. Proulx, M.-E. et Fall, M. « La relève est-elle au rendez-vous au Québec? 》, Fondation de l'entrepreneurship en collaboration avec Caisse de dépôt et placement du Québec, Québec, 2010.
${ }^{2}$ LeBel, L. « Les entrepreneurs forestiers en 2007 : Bilan et un plan d'actions, direction de la recherche PREFORT $», 1^{\text {er }}$ Colloque provincial en entrepreneuriat forestier, Dolbeau-Mistassini, 2007.

${ }^{3}$ Audet, J. «Étude menée auprès des travailleurs forestiers de récolte relativement à leurs aspirations entrepreneuriales ", PReFORT, document de travail no 2008-001, Québec, Université Laval, 2008.

${ }^{4}$ Deschamps, B. et Paturel, R. Reprendre une entreprise... saine ou en difficulté (2nd éd.), Paris, Dunod, 2005.

${ }^{5}$ Cadieux, L. et Brouard, F. La transmission des PME Perspective et enjeux, Québec, Presses de l'Université du Québec, 2009. Hugron, P. " L'entreprise familiale : modèle de réussite du processus successoral », Cahier de recherche, Institut de recherches politiques et Les Presses HEC, 1991.

${ }^{6}$ Malarewicz, J.-A. Affaires de famille; Comment les entreprises familiales gèrent leur mutation et leur succession, Paris, Pearson Education France, 2006. Morris, M.-H., Williams, R.-W. et Nel, D. « Factors Influencing Family Business Succession», International Journal of Entrepreneurial Behavior and Research, vol 2, no 3, 1996, p. 68-81.

${ }^{7}$ Cabrera-Suárez, K. " Leadership Transfer and the Successor's Development in the Family Firm ", The Leadership Quarterly, vol. 16, $\mathrm{n}^{\mathrm{o}}$ 1, 2005, p. 71-96. Cabrera-Suárez, K., De Saá-Pérez, P. et García-Almeida, D. «The succession process from a resource and knowledge based view of the family firm », Family Business Review, vol. 14, n ${ }^{0}$ 1, 2001, p. 37-47. GarciaAlvarez, E., Lopez-Sintas, J. et Gonzalvo, P. S. « Socialization Patterns of Successors in First- to SecondGeneration family businesses », Family Business Review, vol. 15, no 3, 2002, p. 189-205. Gersick K., Davis, J., McCollum-Hampton, M. Lansberg, I. Generation to Generation: Life Cycles of the Family Business, Boston, Harvard Business School Press, 1997.

${ }^{8}$ Richer, F., St-Cyr, L. et Lambaraa, Y. « La transmission d'entreprise au Québec : des stratégies diversifiées ", Gestion, vol 29, no 3, 2004, p. 95-102.

${ }^{9}$ Harrison, R. « Learning and development in the smaller organisation», dans Learning and Development , CIPD Publishing, London, 2002. Sambrook, S. « Exploring Succession Planning in Small, Growing Firms », Journal of Small Business and Enterprise Development, vol 12, $\mathrm{n}^{\circ} 4,2005$, p. 579-594.

${ }^{10}$ Cadieux, L. « Succession in Small and Medium-Sized Family Businesses: Toward a Typology of Predecessor Roles During and After Instatement of the Successor»", Family Business Review, vol. 20, nº 2, 2007, p. 95-109.

${ }^{11}$ Le Breton-Miller, I., Miller, D. et Steier, L. P. « Toward an Integrative Model of Effective FOB Succession ", Entrepreneurship Theory and Practice, vol. 28, $\mathrm{n}^{\circ} 4$, 2004, p. 305-328. Parent, D. et Perrier, J.-P. « "C'est pas juste!" Analyse de l'équité et de la justice dans l'entreprise familiale », Colloque sur l'établissement et le retrait en 
agriculture, Drummondville, 2007, p. 1-14. Senbel, D. «Analyse du transfert de propriété et de son financement au travers 20 cas de relève d'entreprises », dans Cahier de recherche no ${ }^{\circ}$ 06-01, Chaire de développement et de relève de la PME, HEC, Montréal, 2006.

${ }^{12}$ Audet, J. et Couteret, P. « L'accompagnement au service de la relève entrepreneuriale: le cas de la foresterie au Québec ", 9ème CIFEPME, 15 p., Université catholique de Louvain, 2008.

${ }^{13} \mathrm{OSEO}$ « La transmission des petites et moyennes entreprises: l'expérience d'OSEO », OSEO Banque du développement des petites et moyennes entreprises, 2005.

${ }^{14}$ Pinard, R. « La relève entrepreneuriale dans les PME Lavalloises, ça se fait », Laval technopole, Laval, 2008.

${ }^{15}$ Dufour, D. " L'industrie du bois d'œuvre: une contribution cruciale à la prospérité du Canada ", par Division de la fabrication de la construction et de l'énergie (dir.), Statistiques Canada, 2002. Lazar, A. « Améliorer le climat des affaires et la compétitivité canadienne: transcription des remarques d'Avrim Lazar (traduction) ». Forum des politiques publiques, Ottawa, 2007.

${ }^{16}$ Legendre, C. Le travailleur forestier québécois : transformations technologiques, socioéconomiques et organisationnelles, Sainte-Foy, Presses de l'Université du Québec, 2005.

${ }^{17}$ Drolet, S. «L'entrepreneur forestier de récolte, véritable propriétaire-dirigeant de PME? ", 11 Journées scientifiques du Réseau entrepreneuriat, Trois-Rivières, 2009, p. 2-16.

${ }^{18}$ Thiétart, R.-A. et al., Méthodes de recherche en management (3e éd.), Paris, Dunod, 2007.

${ }^{19}$ Yin, R. K. Case Study Research, Design and Methods (end ed.), Newbury Park, Sage, 2003.

${ }^{20}$ Trochim, W. « Research Methods : Knowledge Base», 2002, accès le 12/10/2007, http://www.socialresearch methods.net $/ \mathrm{kb} /$ index.htm.

${ }^{21}$ D'Amboise, G. et Audet, J. La comparaison intersites : une voie pour la recherche en gestion, Québec, Presses de 1'Université Laval, 2005. Miles, M. B. et Huberman, M. Qualitative Data Analysis : A Sourcebook of New Methods, Newbury Park, Sage Publications, 1984.

${ }^{22}$ MAPAQ Portrait de la relève établie 2006-Rapport statistique, par Direction des politiques sur la gestion des risques (dir.), MAPAQ, Québec, 2007.

\section{NOTES}

aFrance Asselin, MBA, présidente Mobius coaching et management.

bÉtienne St-Jean, professeur titulaire, Chaire de recherche UQTR sur la carrière entrepreneuriale, Institut de Recherche sur les PME.

${ }^{c}$ Luc LeBel, professeur titulaire, Faculté de foresterie et de géomatique, Université Laval. 